\title{
Prevalencia e intensidad de infección por geohelmintos, caracterizando los factores socio culturales y ambientales que inciden en la infección de escolares, Paraguay, 2015
}

\author{
Fátima Aidée Vázquez', Dora Rafaela Ramírez', Gloria Echague², Liliana Sosa², \\ María Águeda Cabello',2, Margarita Samudio ${ }^{2}$, Ana Luciañez y Dalva María de Assis ${ }^{4}$
}

Prevalence and intensity of geohelminths infection characterizing the socio-cultural and environmental factors that affect the infection of school children, Paraguay, 2015

Introduction: Currently, Paraguay has no data on the prevalence of soil-transmitted geohelminth infection in schoolchildren aged 6 to 12 years. Aim: To determine the prevalence and intensity of infection by Ascaris lumbricoides, Trichuris trichiura and hookworms and the socio-cultural-environmental characteristics of the affected population. Methods: Descriptive, cross-sectional study, in three semi-tropical climatic zones: continental, semi steppe and humid. A total of 1,404 schoolchildren from 20 schools in eight departments were selected from the country's three climate zones. Copro-parasitological exams were analyzed in situ with the Kato-Katz method. Results: The national prevalence for geohelminthiasis was 3.73\% (95\% CI $=2.8-4.8)$. The risk factors found were consumption of well water OR: $2.88(95 \% \mathrm{CI}=1.64-2.07)$, ground floor in housing OR: $2.56(95 \% \mathrm{CI}=1.45-4.50)$ and lack of flushing water baths in households OR: $2.29(95 \% \mathrm{CI}=1.23-4.28)$. Conclusion: Although the national prevalence of geohelminthiasis was low, it is recommended to promote good hygienic practices, use of footwear, and safe water consumption, as well as designing interventions with all relevant sectors to improve access to safe water and improved basic sanitation facilities.

Keywords: Paraguay; helminthiasis; helminths; prevalence; Ascaris lumbricoides; Trichuris trichiura; hookworms.

Palabras clave: Paraguay; helmintiasis; helmintos; prevalencia; Áscaris lumbricoides; Trichuris trichiura; uncinarias.

\section{Introducción}

egún la Organización Panamericana de la Salud (OPS) y la Organización Mundial de la Salud (OMS), las enfermedades infecciosas desatendidas (EID) son un conjunto de infecciones, muchas de ellas parasitarias, ocasionadas por diversos microorganismos ${ }^{1}$ que afectan principalmente a las poblaciones que viven en condiciones socio-económicas de pobreza y que tienen problemas de acceso a los servicios de salud ${ }^{2}$.

Las geohelmintiasis, o helmintiasis transmitidas por el contacto con el suelo (HTS), son unas de las infecciones prevalentes entre las EID. En el año 2014, 25 países de la región de las Américas tenían como población en riesgo de las HTS cerca de 47 millones de niños de 1 a 14 años, de los cuales 13,3 millones eran pre-escolares y 33,7 millones eran escolares ${ }^{3}$.

Las HTS son un grupo de enfermedades parasitarias causadas por diferentes especies de nematodos que cumplen parte de su ciclo vital en el suelo, donde maduran sus huevos o larvas si las condiciones del suelo les son favorables, haciéndose infectantes y, alcanzando a un nuevo hospedero por vía oral o dérmica. Estos huevos inmaduros están presentes en las heces humanas contaminando el suelo en las zonas con deficientes sistemas de saneamiento. Los niños infectados experimentan deterioro físico, nutricional y cognitivo ${ }^{4}$.

El control de las parasitosis es una tarea compleja. Durante más de dos décadas se han realizado esfuerzos para reducir las geohelmintiasis en América Latina. Entre esos esfuerzos se pueden mencionar: la 54 Asamblea General de la OMS, celebrada en 2001, donde se aprobó una resolución que promueve que estas parasitosis sean consideradas un problema de salud pública ${ }^{5}$; la resolución CD49.R19 con respecto a la eliminación de las EID y otras infecciones relacionadas con la pobreza ${ }^{2}$; la reunión realizada en Colombia, en el año 2013, para intensificar los esfuerzos integrados para el control de las geohelmintiasis trabajando juntos por un objetivo ${ }^{6}$, y el plan de acción para la eliminación de las EID y las medidas posteriores
'Dirección General de Vigilancia de la Salud. Ministerio de Salud Pública y Bienestar Social. Asunción. Paraguay. ${ }^{2}$ Instituto de Investigaciones en Ciencias de la Salud - Universidad Nacional de Asunción. San Lorenzo. Paraguay.

${ }^{3}$ Programa de Enfermedades Infecciosas Desatendidas,

Organización Panamericana de la Salud-Organización Mundial de la Salud, Washington, D.C., Estados Unidos.

${ }^{4}$ Organización Panamericana de la Salud Representación del Paraguay, Asunción. Paraguay.

La presente investigación no presentó conflictos de intereses ni conflictos éticos con ninguna institución, persona o entidad relacionada con el tema. Proyecto fue financiado con el apoyo de: La fundación IZUMI, y por el programa regional de enfermedades infecciosas desatendidas de la OPS/OMS; el proyecto fue implementado por la estructura de salud del Ministerio de Salud Pública y Bienestar Social de Paraguay (MSPBS).

Recibido: 30 de octubre de 2017 Aceptado: 16 de julio de 2018

Correspondencia a: María Águeda Cabello Sarubbi aguedacabello@gmail.com 
a la eliminación en el año 2016-2022 aprobado por OPS en el $55^{\circ}$ Consejo Directivo celebrado en septiembre de $2016^{7}$, entre otros.

En el año 2013 se firmó en Paraguay, un acuerdo entre el Ministerio de Salud Pública y Bienestar Social (MSPBS) y el Ministerio de Educación y Ciencia (MEC) para llevar adelante anualmente la Campaña Nacional de Desparasitación Masiva a Escolares (CNDME), con la cooperación de la OPS/OMS ${ }^{8}$.

La cuantificación adecuada de la morbilidad asociada a las geohelmintiasis y de la carga de enfermedad es fundamental para diseñar e implementar programas de desparasitación a gran escala. Con el objetivo de direccionar las campañas de desparasitación masiva, la OPS/ OMS recomienda los estudios de prevalencia parasitaria por medio de copro-parasitología, mediante el método de Kato-Katz, el que determina el número de huevos de geohelmintos por gramo de heces y permite establecer la intensidad de infección parasitaria, pudiendo así clasificarlas en grados de infección: leves, moderadas o intensas ${ }^{9-12}$. Hasta el año 2015, Paraguay no contaba con una línea de base o un estudio de prevalencia y distribución de los geohelmintos en el país, que pudiera direccionar las acciones de prevención y control de la geohelmintiasis.

El objetivo principal de este estudio fue determinar la prevalencia e intensidad de geohelmintiasis en niños en edad escolar en Paraguay, pues se sabe que este resultado ayudará para la toma de decisiones por parte del MSPBS en cuanto a las actividades que involucren la desparasitación masiva en el país. Los objetivos específicos fueron caracterizar algunos factores socio-demográficos presentes en los escolares, analizar la asociación estadística de las medidas antropométricas y los factores de riesgos socioeconómicos y medioambientales en relación a las geohelmintiasis.

\section{Materiales y Métodos}

\section{Definición del universo y población de estudio}

Se tomó en cuenta todas las escuelas del país. La población foco fueron los escolares de 6 a 12 años de edad que cursaban desde el primero hasta el sexto grado (incluidos los escolares de preescolar y séptimo grado que cumplían con la edad de 6 y 12 años, respectivamente) de las escuelas que fueron incluidas en el estudio. Se utilizaron informaciones provistas por el MEC correspondiente a los datos del censo de escuelas del año $2010^{13}$.

\section{Tipo de estudio}

Estudio descriptivo, de corte transversal, con componente analítico. El territorio nacional fue estratificado por zonas climáticas: semi tropical continental, semi tropical semi estépico y semi tropical húmedo ${ }^{14}$ y por sector (urbano y rural).

\section{Tamaño muestral}

Para la estimación del número de alumnos a ser incluidos en cada uno de los estratos, se consideró el tamaño de la población de cada estrato y la prevalencia encontrada en estudios similares a lo largo de América. Por lo tanto, se utilizó una prevalencia de $10 \%$, un efecto de diseño de 1,5 , error absoluto de $5 \%$ y el error alfa de 0,05 . Al resultado se agregó $10 \%$ por probable ausentismo, rechazo para participar en el estudio, falta de muestras o muestras inadecuadas de heces. El cálculo se realizó utilizando EpiInfo 7 (CDC, Atlanta). La distribución de la muestra calculada a nivel nacional por zona climática fue de la siguiente forma: Semi tropical continental 415 niños, semitropical semi estépico 456 niños y semi tropical húmedo 456 niños; representando un total de 1.327 niños a reclutar.

\section{Criterios de inclusión}

Escolares que tienen entre 6 y 12 años de edad y con consentimiento del padre o tutor y consentimiento por parte del niño(a) para la recolección de la muestra y medición antropométrica.

\section{Criterios de exclusión}

Niño(a) que en el momento del operativo de campo se encuentre ausente o que su padre o tutor no autorice a participar del estudio.

\section{Procedimiento de muestreo}

Primera etapa: Definición de estratos y de entidades geográficas. Se realizó un muestreo multi-etápico estratificado en zonas climáticas: semi tropical continental (departamentos de Alto Paraguay y Boquerón), semi tropical semi estépico (departamentos de Presidente Hayes, Concepción, San Pedro, Cordillera, Central, Amambay, Canindeyú y Caaguazú), semi tropical húmedo (departamentos de Alto Paraná, Itapúa, Guairá, Caazapá, Misiones, Neembucú y Paraguarí) y por sector rural y urbano.

Para la división por zona climática y sector (urbana y rural) se consideraron las características del suelo, la presencia de agua y las condiciones geográfico climáticas ${ }^{15,16}$, que se relacionan con el ciclo de vida del parásito y su etapa de maduración en el suelo, que pueden influir en las posibles diferencias de las prevalencias encontradas.

Segunda etapa: Selección de las escuelas y niños. Una vez distribuidas las escuelas por zona climática y sector, se creó un listado con los grados (cohortes) de interés de cada estrato; luego, las escuelas fueron ordenadas según la cantidad de alumnos de los grados objeto de estudio y de menor a mayor por el número total de alumnos de cada escuela.

Se excluyeron las escuelas con un número de alumnos 
inferior al cuartil $1(25 \%)$ y los superiores al cuartil 3 (75\%). Dentro de las escuelas incluidas se realizó un sorteo aleatorio, seleccionando para cada zona un número de escuelas que permita tener el número de alumnos necesarios para alcanzar el tamaño de muestra.

Una vez distribuidas las escuelas por zona ecológica y sector, se creó un listado con los grados de interés de cada estrato, luego las escuelas fueron ordenadas según la cantidad de alumnos de los grados objeto de estudio $\mathrm{y}$ de menor a mayor por el número total de alumnos de cada escuela.

Para la encuesta nacional fueron objeto de estudio 20 escuelas de ocho departamentos del país. En relación a la zona climática, ocho escuelas pertenecían a la zona climática semi tropical continental; seis escuelas a la zona climática semi tropical semi estépico y seis escuelas a la zona climática semi tropical húmedo (Figura 1).

\section{Período de estudio}

El estudio fue realizado en los meses de agosto y septiembre del año 2015, considerando que la CNDME es realizada en Paraguay en el inicio del segundo semestre (entre julio y agosto de cada año), por lo tanto, fue acordado con las zonas de las escuelas participantes (seleccionadas en el muestreo) que solamente iniciará la CNDME y distribuciones de los antiparasitarios en los escolares después de la realización del estudio.

\section{Procedimiento de laboratorio y recolección de información}

Siguiendo la recomendación de la OMS, la investigación parasitaria se hizo por medio de copro-parasitología, utilizando el método de concentración (Kato-Katz). Esta técnica permitió el recuento de los huevos por especie en toda la preparación. La preparación se examinó en forma sistemática y se anotó el resultado de cada cuenta individual por especie, lo que permitió clasificar el nivel de infección por tipo de helmintos. Se debe evitar que el tiempo de aclaración se exceda para no dificultar la observación de los huevos, garantizando la confiabilidad analítica.

Para determinar la intensidad de infección expresada en huevos por gramo de heces, el número de huevos de cada una de las especies de helmintos se multiplica por 24 (en cada muestra hay $41,7 \mathrm{mg}$ de heces). La magnitud se clasifica en leve, moderada o intensa según el cuadro de interpretación de recuento de huevos de geohelmintos ${ }^{10}$. Se montó un laboratorio in situ, debido a la distancia de las escuelas seleccionadas para analizar las muestras en fresco.

En relación al control de calidad, sólo la práctica de la técnica durante varias veces ayudó a establecer los tiempos específicos para cada espécimen y la concordancia entre observadores.

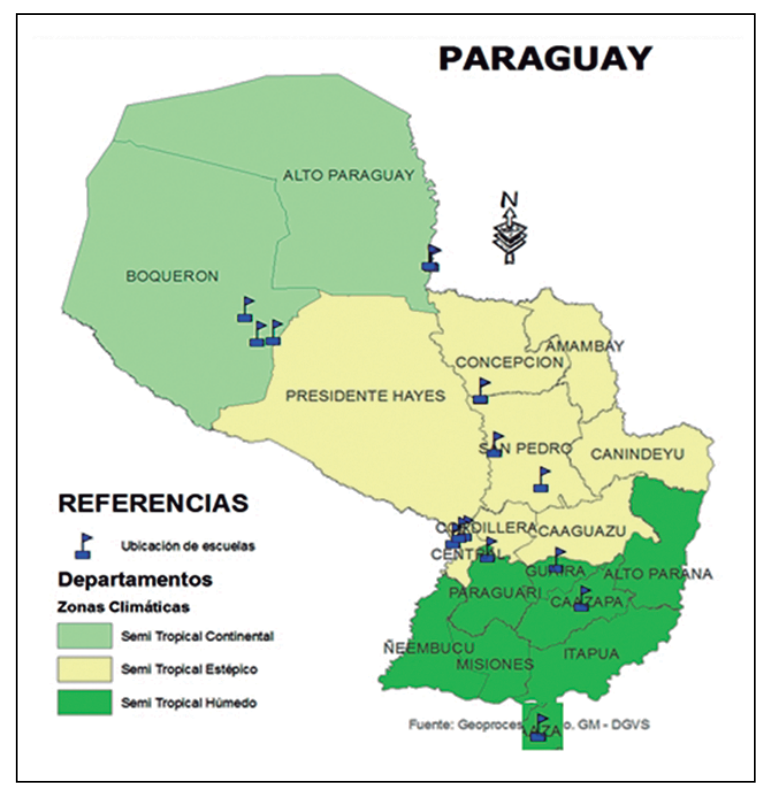

Figura 1. Distribución geográfica de las escuelas.

\section{Medición de las variables}

Para el levantamiento de la información se conformó un equipo de trabajo debidamente capacitado, quienes aplicaron dos encuestas. Una dirigida a los padres, madres y/o tutores para la recolección de la siguiente información: datos socio demográficos, datos de los padres, madres y/o tutores, características de las viviendas, suministro de agua, servicios sanitarios dentro de la vivienda, tratamiento de las basuras, cuando fue la última vez que el niño tomó antiparasitario y quien le proveyó, uso de calzados. Otra encuesta dirigida a los directores de la institución para obtener datos socio-demográficos, censo de alumnos de la institución, suministro de agua, eliminación de los desechos sólidos, servicios higiénicos dentro de la escuela. Y un formulario para los niños que constaba de lo siguiente: datos socio-demográficos, datos del niño (sexo y edad), estado nutricional y resultados de laboratorio. Al mismo tiempo se tomaron las medidas antropométricas de los escolares utilizando una balanza electrónica para la medición del peso y un tallímetro para la medición de la altura. Para el cálculo del estado nutricional se utilizó el gráfico nutricional de la OPS/OMS del año $2007^{17}$.

\section{Análisis estadístico}

Se utilizó el programa informático EpiInfo 7 $7 \mathrm{TM}$ (CDC, Atlanta) y para la elaboración de gráficos Microsoft Excel 2010. Para el análisis se utilizaron medidas estadísticas de tendencia central y dispersión, número absoluto, frecuencias y tasas con sus intervalos de confianza de $95 \%$ (IC 95\%). Se calculó la Odds Ratio (OR) como medida de asociación. Las pruebas estadísticas de base fueron el $\chi^{2}$ y Test Exacto de Fisher según correspondiera para comparar proporciones y prueba t-student para comparar 
medias, considerándose significativa el valor de probabilidad $\mathrm{p} \leq 0,05$.

Para el cálculo de la prevalencia de geohelmintiasis causada por cualquiera de los helmintos se estimó con base en las infecciones individuales, utilizando el método descrito por Silva y Hall donde la prevalencia para cada helminto fue colectada de forma separada, y mediante un método matemático fue estimada la prevalencia total a partir de la prevalencia de cada helminto ${ }^{18}$. Además, fue calculada la intensidad de la infección de acuerdo al número de helmintos que infectan a cada escolar clasificándola en leve, moderada e intensa.

\section{Aspectos éticos}

Este protocolo fue sometido al Comité de Ética de la OPS/OMS donde fue aprobado por la PAHOERC Ref. $\mathrm{N}^{\circ}$ : PAHO-2014-11-0032. Además, fue aprobado por el Comité Ético del Instituto de Investigaciones en Ciencias de la Salud (IICS), con número de código P15/2014.

Los padres o tutores responsables de los escolares de los grados seleccionados firmaron un consentimiento informado y los escolares el asentimiento. Los resultados fueron entregados a los padres o tutores y los niños fueron remitidos y asistidos por el equipo médico del Centro de Salud local, y se les proveyó del antiparasitario albendazol, $400 \mathrm{mg}$ diarios por vía oral durante cinco días en caso de positivo para helmintos y metronidazol $20 \mathrm{mg} / \mathrm{kg} / \mathrm{día}$ durante 7 días en el caso de que saliera resultado positivo para protozoario, ya que el estudio abordó otros tipos de parásitos, resaltando que estos parásitos no helmintos no fue objeto de estudio para esta investigación.

Adicionalmente, al final de la intervención en las estaciones de trabajo, los escolares recibieron tratamiento antiparasitario para geohelmintos, con una dosis única de albendazol en tabletas masticables de $400 \mathrm{mg}$.

\section{Resultados}

Fueron sujetos de estudio 1.617 escolares y se analizaron muestras de 1.404 escolares (87\% del tamaño estimado de la muestra). Entre 15,7 y 16,2\% de los niños se encontraban en la franja etaria de 9 a 11 años.

La prevalencia total de geohelmintiasis fue de 3,7\% $\mathrm{IC}_{95 \%}(2,8-4,8)$, observándose diferencias significativas de acuerdo a la zona climática. En la zona semi tropical húmedo la prevalencia fue de 5,7\%; en el sector rural fue $4,4 \% \mathrm{IC}_{95 \%}(3,2-6,1)$ y en el urbano $2,8 \% \mathrm{IC}_{95 \%}(1,7-4,4)$. La prevalencia por helmintos para Ascaris lumbricoides fue de $1,9 \%$ y para uncinarias $1,7 \%$. No se registraron casos de Trichuris trichiura. Se observó una prevalencia de 4,7\% IC95\% $(2,3-8,5)$ en los escolares de ocho años. La prevalencia para el sexo femenino fue $4,1 \% \mathrm{IC}_{95 \%}(2,8-$ $5,9)$ y para el masculino $3,3 \% \mathrm{IC}_{95 \%}(2,2-4,8)$, diferencia no significativa (Tabla 1 ).

\begin{tabular}{|c|c|c|c|c|c|c|c|c|}
\hline & n & $\%$ & $\begin{array}{l}\text { n de positivos para } \\
\text { Á. lumbricoides }\end{array}$ & $\%$ & $\begin{array}{l}\mathrm{n} \text { de positivos } \\
\text { para uncinarias }\end{array}$ & $\%$ & & $\begin{array}{l}\text { valencia } \\
\text { C (95\%) }\end{array}$ \\
\hline \multicolumn{9}{|l|}{ Zona climática } \\
\hline Semi tropical continental & 476 & 33,90 & 2 & 0,42 & 4 & 0,84 & 1,26 & $(0,5-2,6)$ \\
\hline Semi tropical semi estépico & 465 & 33,12 & 16 & 3,44 & 4 & 0,86 & 4,27 & $(2,7-6,4)$ \\
\hline Semi tropical húmedo & 463 & 32,98 & 10 & 2,16 & 17 & 3,67 & 5,75 & $(3,9-8,2)$ \\
\hline \multicolumn{9}{|l|}{ Sector } \\
\hline Rural & 776 & 55,27 & 14 & 1,80 & 21 & 2,70 & 4,46 & $(3,21-6,14)$ \\
\hline Urbano & 628 & 44,73 & 14 & 2,22 & 4 & 0,52 & 2,85 & $(1,76-4,40)$ \\
\hline \multicolumn{9}{|l|}{ Edad (años) } \\
\hline 6 & 157 & 11,18 & 5 & 3,18 & 2 & 1,27 & 4,41 & $(1,9-8,6)$ \\
\hline 7 & 175 & 12,46 & 3 & 1,71 & 5 & 2,86 & 4,52 & $(2,1-8,4)$ \\
\hline 8 & 189 & 13,46 & 7 & 3,70 & 2 & 1,06 & 4,72 & $(2,3-8,5)$ \\
\hline 9 & 221 & 15,74 & 3 & 1,36 & 4 & 1,81 & 3,14 & $(1,3-6,1)$ \\
\hline 10 & 225 & 16,03 & 3 & 1,33 & 4 & 1,78 & 3,09 & $(1,3-6,0)$ \\
\hline 11 & 228 & 16,24 & 6 & 2,63 & 3 & 1,32 & 3,92 & $(1,9-7,1)$ \\
\hline 12 & 209 & 14,89 & & 0,48 & 5 & 2,39 & 2,86 & $(1,1-5,8)$ \\
\hline \multicolumn{9}{|l|}{ Sexo } \\
\hline Femenino & 689 & 49,07 & 18 & 2,61 & 11 & 1,60 & 4,17 & $(2,8-5,9)$ \\
\hline Masculino & 715 & 50,93 & 10 & 1,40 & 14 & 1,96 & 3,33 & $(2,2-4,8)$ \\
\hline Total & 1.404 & 100 & 28 & 1,99 & 25 & 1,78 & 3,73 & $(2,8-4,8)$ \\
\hline
\end{tabular}




\begin{tabular}{|c|c|c|c|c|c|c|c|c|}
\hline Estado Nutricional & $\begin{array}{l}\text { Semi tropical } \\
\text { continental } \\
n=476\end{array}$ & $\begin{array}{l}\% \\
34\end{array}$ & $\begin{array}{l}\text { Semi tropical } \\
\text { Semi estépico } \\
\qquad n=465\end{array}$ & $\begin{array}{l}\% \\
33\end{array}$ & $\begin{array}{l}\text { Semi tropical } \\
\text { húmedo } \\
n=463\end{array}$ & $\begin{array}{l}\% \\
33\end{array}$ & $\begin{array}{c}\text { Total } \\
\mathrm{n}=1.404\end{array}$ & $\begin{array}{c}\% \\
100\end{array}$ \\
\hline Obesidad & 71 & 14,9 & 40 & 8,6 & 50 & 10,8 & 161 & 11,5 \\
\hline Sobrepeso & 117 & 24,6 & 97 & 20,9 & 89 & 19,2 & 303 & 21,6 \\
\hline Adecuado & 277 & 58,2 & 287 & 61,7 & 306 & 66,1 & 870 & 61,9 \\
\hline Riesgo de desnutrición & 10 & 2,1 & 37 & 8,0 & 16 & 3,5 & 63 & 4,5 \\
\hline Desnutrición & 1 & 0,2 & 4 & 0,9 & 2 & 0,4 & 7 & 0,5 \\
\hline
\end{tabular}

\begin{tabular}{|c|c|c|c|c|c|c|c|}
\hline \multirow[t]{2}{*}{ Características } & Total (\%) & $\begin{array}{c}\text { Semi tropical } \\
\text { continental }\end{array}$ & $\%$ & $\begin{array}{l}\text { Semi tropical } \\
\text { estépico }\end{array}$ & $\%$ & $\begin{array}{l}\text { Semi tropical } \\
\text { húmedo }\end{array}$ & $\%$ \\
\hline & $1.061(100)$ & 388 & 37 & 361 & 34 & 312 & 29,4 \\
\hline \multicolumn{8}{|l|}{ Fuente de agua en la vivienda } \\
\hline ESSAP o Junta de saneamiento & $316(29,8)$ & 2 & 0,5 & 119 & 32,9 & 195 & 62,5 \\
\hline Aguateria & $165(15,6)$ & 5 & 1,3 & 154 & 42,7 & 6 & 1,9 \\
\hline Pozo común* & $53(5)$ & 2 & 0,5 & 9 & 2,5 & 42 & 13,4 \\
\hline Pozo artesiano & $118(11,1)$ & 6 & 1,5 & 54 & 14,9 & 58 & 18,6 \\
\hline Tanque de agua & $56(5,3)$ & 26 & 6,7 & 25 & 6,9 & 5 & 1,6 \\
\hline Otros** & $343(33,3)$ & 347 & 89 & 0 & 0 & 6 & 1,9 \\
\hline \multicolumn{8}{|l|}{ Sanitario } \\
\hline Con arrastre de agua, cámara séptica y pozo ciego & $494(46,5)$ & 79 & 20 & 210 & 58,2 & 205 & 65,7 \\
\hline Letrina & $565(53,2)$ & 309 & 79,6 & 150 & 41,5 & 106 & 34 \\
\hline Otros (baño comunitario, arroyo, río, suelo en el patio, etc.) & $2(0,2)$ & 0 & 0 & 1 & 0,3 & 1 & 0,3 \\
\hline \multicolumn{8}{|l|}{ Desechos de residuos } \\
\hline Quema & $769(72,5)$ & 303 & 78 & 272 & 75,3 & 194 & 62,1 \\
\hline Entierra & $123(11,6)$ & 56 & 14 & & 10,5 & 29 & 9,3 \\
\hline Lleva el camión recolector & $127(11,9)$ & 1 & 0,3 & & 12,2 & 82 & 26,3 \\
\hline Otros*** & 42 (4) & 28 & 7,2 & & 2 & 7 & 2,2 \\
\hline
\end{tabular}

En relación a la intensidad de la infección de los geohelmintos encontrados se observó una mediana de 1.260 huevos por gramo de heces con un rango de 24 a 22.728 para $A$. lumbricoides y 360 con un rango de 48 a 8.376 para uncinarias. La intensidad fue leve para ambos helmintos.

El $61,9 \%$ de los escolares tenía un estado nutricional adecuado, la obesidad fue de $11,5 \%$ y sobrepeso $21,6 \%$; el 5,0\% presentaba riesgo de desnutrición o desnutrición. Tres escolares parasitados con Á. lumbricoides y tres escolares parasitados con uncinarias presentaron riesgo de desnutrición (Tabla 2).

El 29,8\% de las personas consume agua de la Empresa de Servicios Sanitarios de Paraguay S.A (ESSAP) o junta de saneamiento. El 46,5\% poseen servicio sanitario con arrastre de agua, cámara séptica y pozo ciego, y 72,5\% disponen de su basura quemándola (Tabla 3).

En cuanto a las características de las escuelas: $35,0 \%$ bebe agua de pozo y $15,0 \%$ bebe de la red de agua potable. Cuarenta y cinco por ciento de escuelas sólo cuentan con letrinas y 75,0\% elimina sus desechos por medio de la quema (Tabla 4).

Los factores de riesgos asociados a una mayor posibilidad de infección por geohelmintos con A. lumbricoides y uncinarias fueron: el consumo de agua de pozo (OR: 2,88; IC95\% = 1,64-5,08), tener casa con piso de tierra (OR: 2,56 ; IC $95 \%=1,45-4,50$ ) y no contar con un sistema de desagüe de excretas. No se encontró asociación en relación al sexo, al consumo de agua potable y al uso o no de calzado de forma permanente (Tabla 5). 


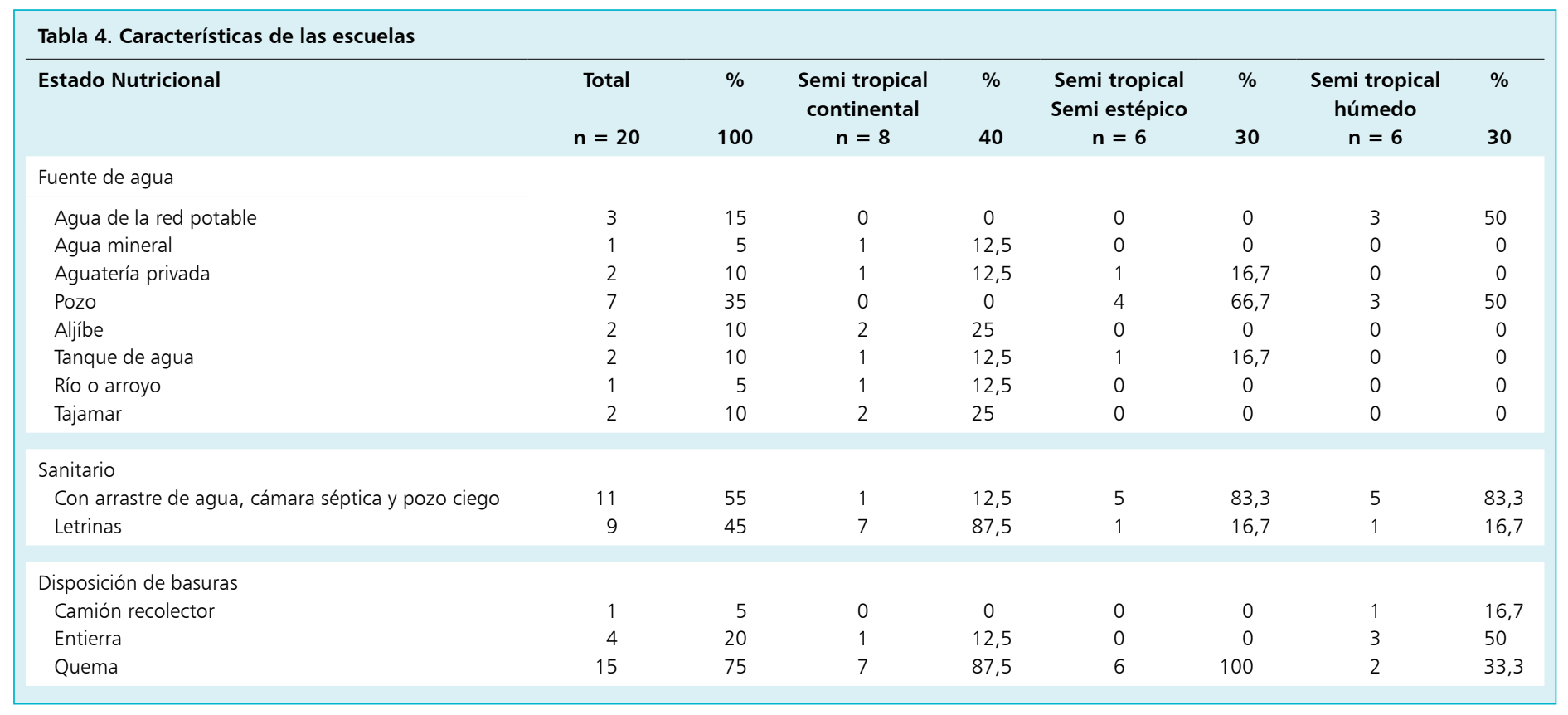

\begin{tabular}{|c|c|c|c|c|}
\hline \multirow[t]{2}{*}{ Factores de riesgo } & & \multicolumn{2}{|c|}{ Geohelmintos } & \multirow[t]{2}{*}{$\mathrm{OR}^{*}(\mathrm{IC} 95 \%)^{* *}$} \\
\hline & & Sí & No & \\
\hline \multirow[t]{2}{*}{ Consumo de agua de pozo } & Sí & 29 & 424 & \multirow{2}{*}{$2,88(1,64-5,08)$} \\
\hline & No & 22 & 929 & \\
\hline \multirow[t]{2}{*}{ Tener piso de tierra } & Sí & 23 & 329 & \multirow[b]{2}{*}{$2,56(1,45-4,50)$} \\
\hline & No & 28 & 1.024 & \\
\hline \multirow{2}{*}{$\begin{array}{l}\text { No contar con un sistema de } \\
\text { desague de excretas }\end{array}$} & Sí & 37 & 724 & \multirow[b]{2}{*}{$2,29(1,23-4,28)$} \\
\hline & No & 14 & 629 & \\
\hline
\end{tabular}

\section{Discusión}

La prevalencia total de geohelmintos en escolares de 6 a 12 años en Paraguay encontrada en este estudio fue de $3,7 \%$ que, de acuerdo con los criterios de la OMS/ OPS, corresponde a un bajo riesgo de infección, por encontrarse por debajo de $20 \%$ de prevalencia ${ }^{18,19}$. Así mismo, cuando se evaluó la intensidad de la infección por los geohelmintos, en la mayoría de los casos positivos la infección fue de leve intensidad.

En este estudio se evidenció que la zona semi tropical húmeda presentó la mayor prevalencia de los geohelmintos estudiados; sin embargo, corresponde a un bajo riesgo de infección ${ }^{18,19}$. Las prevalencias fueron similares a la encontrada en el estudio realizado en escuelas, por Zunini M. y cols. en el año 2001 en un distrito de la zona semi tropical húmeda del país ${ }^{20}$. Cabe destacar que ni en el mencionado estudio, ni en el presente se pudo observar la presencia de $T$. trichiura.

En un estudio realizado en El Chaco en el año 1998 por Mayans en el Departamento de Presidente Hayes ${ }^{21}$ tampoco se encontró la presencia de T. trichiura.

Canese y cols. ${ }^{22}$, observaron este mismo patrón en cinco departamentos pertenecientes a las zonas climáticas tropical semi estépico y semi húmedo en la década del 90. Teniendo en cuenta que Paraguay es un país en vías de desarrollo y con características de saneamientos básicos precarios, se esperaría que la prevalencia de estos parásitos fuera más alta. Sin embargo, en otros estudios locales realizados en los años 1997 y 2015 la prevalencia de los helmintos (A. lumbricoides y uncinarias) fue también menos de $20 \%{ }^{23,24}$

Como factores de riesgo se encontró que los escolares que consumían agua de pozo, tenían piso de tierra en la casa y no contaban con un sistema de disposición de excretas tuvieron una mayor probabilidad de estar parasitados por geohelmintos. Estas tres condiciones de saneamiento básico precario favorecen la infección de la población en estudio. En un estudio realizado recientemente en El Salvador se analizaron algunas variables similares, coincidiendo una asociación con el consumo de agua de pozo $^{25}$. 
No hubo asociación entre los escolares parasitados y la desnutrición, lo que sí fuera observado en otro estudio realizado en Argentina ${ }^{24}$; más de $80 \%$ de los niños con parásitos se encontraban dentro del rango de una nutrición adecuada.

En cuanto a las condiciones de las escuelas se observó que una gran cantidad de ellas no están abastecidas con agua potable, exponiendo así a los escolares al consumo de agua insegura, teniendo en cuenta esto como factor de riesgo asociado a la infección por geohelmintos. $\mathrm{La}$ misma situación fue observada en el porcentaje de letrinas utilizadas en ciertas regiones identificadas.

Los protocolos de desparasitaciones masivas que se realizan anualmente en el país deberían ser revisados ya que, en base a los hallazgos del presente estudio, la prevalencia es $>1 \% \mathrm{y}<10 \%$, es decir de bajo riesgo de infección, por lo cual los estándares internacionales sugieren realizar quimioterapia preventiva antihelmíntica una vez cada dos años ${ }^{18,19}$. Sin embargo, las condiciones de agua y saneamiento no son satisfactorias corriéndose el riesgo de que la quimioprofilaxis cada dos años no sea suficiente.

Las campañas de desparasitación masiva que se vienen realizando en el país han contribuido a tener bajos valores de prevalencia por geohelminto e intensidad leve de infección. Sin embargo, teniendo en cuenta que las condiciones de agua y saneamiento no son satisfactorias, es necesario continuar realizando la quimioterapia preventiva antihelmíntica, y a la vez, trabajar intersectorialmente para mejorar las condiciones de agua y saneamiento. Así como la educación sobre higiene personal y ambiental. Actualmente el MSPBS está elaborando un plan integrado de Control y Eliminación de las Enfermedades Infecciosas Desatendidas, que contempla el control de las geohelmintiasis mediante el tratamiento preventivo de las mismas a través de campañas de desparasitación, haciendo énfasis en un manejo intersectorial de los factores determinantes de éstas, y especialmente los que tienen que ver con el acceso a una vivienda adecuada, agua segura y saneamiento básico, con énfasis en la disposición adecuada de excretas y la educación.

Agradecimientos. A todos los docentes de las escuelas, a los escolares que participaron, a las personas y entidades del equipo de salud que participaron durante todas las etapas de la investigación como así también al equipo del laboratorio de la Universidad Nacional de Asunción.

\section{Resumen}

Introducción: Actualmente Paraguay no cuenta con estudios de prevalencia de infección por geohelmintos transmitidos por contacto con el suelo en escolares de 6 a 12 años. Objetivo: Determinar la prevalencia e intensidad de infección por Ascaris lumbricoides, Trichuris trichiura y las uncinarias y las características socio culturalesambientales de la población afectada. Materiales y Métodos: Estudio descriptivo, de corte transversal, en tres zonas climáticas: semi tropical continental, semi tropical semi estépico y semi tropical húmedo. Se seleccionaron 1.404 escolares de 20 escuelas de ocho departamentos, de las tres zonas climáticas del país. Se analizaron exámenes coproparasitológicos in situ con el método Kato-Katz. Resultados: La prevalencia nacional de geohelmintiasis fue de $3,7 \%$ (IC95\% $=2,8-4,8)$. Los factores de riesgo fueron el consumo de agua de pozo OR: $2,88($ IC95\% $=1,64-2,07)$, piso de tierra en la vivienda OR: 2,56 (IC95\% $=1,45-$ $4,50)$ y falta de baño con arrastre de agua en los hogares OR: 2,29 (IC95\% = 1,23-4,28). Conclusión: A pesar de que la prevalencia nacional de geohelmintiasis fue baja, se recomienda promocionar buenas prácticas higiénicas, el uso de calzado, y consumo de agua segura, así como diseñar intervenciones con los sectores pertinentes para mejorar el acceso al agua segura y a instalaciones mejoradas de saneamiento básico.

\section{Referencias bibliográficas}

1.- Organización Panamericana de la Salud Organización Mundial de la Salud. Resolución CD49.R19 Eliminación de las Enfermedades Desatendidas y otras Infecciones Relacionadas con la Pobreza. [Internet] 2009 [consultado el 28 de diciembre de 2016]. Disponible en: http:// www.paho.org/hq/dmdocuments/2009/CD49. R19\%20\%28Esp.\%29.pdf

2.- Organización Panamericana de la Salud - Organización Mundial de la Salud. Enfermedades Infecciosas Desatendidas y otras enfermedades relacionadas con la pobreza en las Américas. [Internet] 2009 [consultado el
7 de noviembre de 2014]. Disponible:http:// www.paho.org/pahobranding/wpcontent/ uploads/2013/08/EID_Opt1_v12.pdf

3.- Organización Panamericana de la SaludOrganización Mundial de la Salud. Geohelmintiasis. [Internet] 2014 [consultado el 3 de marzo del 2017]. Disponible en: http:// www.paho.org/hq/index.php?option=com_topic s\&view $=$ article\&id=419\&Itemid $=41001 \&$ lang $=\mathrm{es}$

4.- Organización Panamericana de la SaludOrganización Mundial de la Salud. Helmintiasis transmitida por el suelo. [Internet] 2017 [actualizado setiembre del 2017]. [consultado el 30 de septiembre de 2017]. Disponible en: http://www.who.int/mediacentre/factsheets/ fs $366 /$ es/

5.- Organización Panamericana de la Salud Organización Mundial de la Salud. Resolución WHA54.19 Esquistosomiasis y helmintiasis transmitidas por el suelo. [Internet] 2001 [consultado el 3 de marzo de 2017]. Disponible: http://www.who.int/neglected_diseases/ mediacentre/WHA_54.19_Esp.pdf?ua=1

6.- Organización Panamericana de la Salud Organización Mundial de la Salud. Reunión para intensificar los esfuerzos integrados para el control de las geohelmintiasis en la Región de las Américas, trabajando juntos por un mismo objetivo Bogotá, Colombia, 13 al 17 
de mayo de 2013. [Internet] 2013 [consultado el 3 de marzo de 2017]. Disponible en: http:// www.paho.org/hq/index.php?\%20option=com 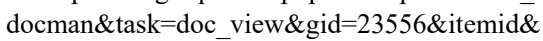 Itemid $=270$

7.- Organización Panamericana de la SaludOrganización Mundial de la Salud. Plan de Acción para la Eliminación de las Enfermedades Infecciosas Desatendidas y las medidas posteriores a la eliminación 20162022. [Internet] 2016 [consultado el 3 de marzo de 2017] Disponible en: http://www.paho.org/ hq/index.php?option $=$ com_docman\&task $=$ doc view\&gid=35740\&Itemid $=270 \&$ lang $=$ es.

8.- Ministerio de Salud Pública y Bienestar Social. Guía de la Campaña Nacional de Desparasitación Masiva a Escolares Paraguay 2015. [Internet] 2015 [consultado el 3 de marzo de 2017]. Disponible en: http://iris.paho.org/xmlui/bitstream/ handle/123456789/10111/Guia\%20CNDME. pdf?sequence $=1$ \&isAllowed $=y$

9.- World Health Organization. Diagnostic Techniques for Intestinal Parasitic (IPI) Applicable to Primary Health Care (PHC) Services. [Internet] 1986 [consultado el 5 de mayo de 2014] Disponible en: http://libdoc. who.int/hq/1985-86/PDP_85.2.pdf

10.- Katz N, Chaves A, Pellegrino J. A simple device for quantitative stool thick-smear technique in Schistosomiasis mansoni. Rev Inst Med Trop São Paulo 1972;14 (6): 397-400.

11.- World Health Organization. Cellophane faecal thick smear examination technique (Kato) for diagnosis of intestinal schistosomiasis and gastrointestinal helminth infections [Internet]. [consultado el 5 de mayo de 2014]. Disponible en: http://www.sci.unal.edu.co/ scielo.php?script $=$ sci_arttext\&pid $=\mathrm{S} 0120$ 41572013000100016\&lng=en\&nrm=iso\&tlng= es.

12.- Organización Panamericana de la Salud. Manual de Manejo de Enfermedades Parasitarias Prioritarias en Honduras. [Internet]
2009 [consultado el 5 de mayo de 2014]. Disponible en: http://www.bvs.hn/Honduras/ IAV/Manual\%20IAV\%202009.pdf

13.- Ministerio de Educación y Cultura. Listado de instituciones de educación inicial y escolar básico año 2010. [consultado el 7 de noviembre de 2014].

14.- Papadakis J. 1980. El clima; Con especial referencia a los climas de América Latina, Península Ibérica, Ex colonias Ibéricas, y sus potencialidades agropecuarias. 377 p. Editorial Albatros. [consultado el 7 de noviembre de 2014].

15.- PARASITOLOGÍA - Triada Ecológica. [Internet] 2009 [consultado el 7 de noviembre de 2014]. Disponible en: http://unesparasitologia.es.t1/Triada-Ecol\%F3gica.htm

16.- Pumarola A, Rodríguez-Torres J A, García-Rodríguez J A, Piedrola-Ángulo G. Microbiología y Parasitología Médica. $2^{\mathrm{a}} \mathrm{ed}$. Madrid: Silvat. P 877.

17.- Organización Mundial de la Salud. Referencia de crecimiento 5-19 años. [Internet]. [consultado el 7 de noviembre de 2014]. Disponible en: http://www.who.int/growthref/ who2007_bmi_for_age/en/

18.- World Health Organization. Helminth control in school-age children: A guide for managers of control programmes. 2nd edition. Geneva: WHO; 2011.

19.- Ministerio de Salud y Protección Social. Lineamientos de desparasitación antihelmíntica masiva, en el marco de la estrategia "quimioterapia preventiva antihelmíntica de OMS". Colombia, 2013. [Internet]. [consultado 03 de marzo del 2017]. Disponible en: http://www.ins.gov. co/normatividad/Lineamientos\%20Sector/ LINEAMIENTO\%20DESPARASIT. $\% 20$ ANTIHELM $\%$ C3\%8DNTICA $\% 20$ MASIVA $\% 20$ $\%$ E2\%80\%9CQUIMIOTERAPIA $\% 20$ PREVENTIVA $\% 20$ ANTIHELM $\%$ C3\%8DNTICA $\% 20$ DE $\% 20$
OMS $\%$ E2 $\% 80 \% 9$ D.pdf?Mobile $=1 \&$ Source $=\%$ 2Fnormatividad\%2F\%5Flayouts\%2Fmobile $\% 2$ Fview\%2Easpx\%3FList\%3D9a3eeed1\%252Da db1\%252D4363\%252D8458\%252Da3cd9d2b4 $835 \% 26$ View\%3D355f8e3f\%252D6790\%252D $4274 \% 252 \mathrm{Db} 220 \% 252 \mathrm{De} 120 \mathrm{c} 0705 \mathrm{df} \%$ \%26ur rentPage $\% 3 \mathrm{D} 1$

20.- Zunini M, Núñez M T. Prevalencia de las parasitosis intestinales en escolares a nivel de la comunidad; Prevalence of intestinal parasitosis in scholashipsoncommunitylevel. [Internet]. [consultado el 7 de noviembre de 2014] Disponle en: http://bases.bireme.br/cgibin/ wxislind.exe/iah/online/?IsisScript=iah/iah.xis $\& \mathrm{src}=$ google $\&$ base $=$ LILACS\&lang $=\mathrm{p} \&$ nextA ction=lnk\&exprSearch=390102\&indexSearch= ID.

21.- Mayans N, Calderoli I, Cabello E, Canese A. Parasitosis intestinal. XIV Congreso Latinoamericano de Microbiologia 1998. página 90 .

22.- Canese A, Barrios E, Castro L, Canese L. Prevalencia de parásitos intestinales en niños del Paraguay. Rev. Parag. Microbiol 1999; 1 (19): 14-8

23.- Pistilli N, Melgarejo M, Ramírez A, Laviosa R, Sosa L. Parasitosis intestinal y anemia en escolares de zonas aledañas al río Paraguay en Asunción. Ann Rep 1997; 39-43.

24.- Echague G, Sosa L, Días V, Ruíz I, Rivas Lourdes, Granado D, et al. Enteroparasitosis en niños bajo 5 años de edad, indígenas y no indígenas, de comunidades rurales del Paraguay. Rev. Chilena Infectol 2015; 32 (6): 649-57. http://dx.doi.org/10.4067/S071610182015000700006.

25.- Sorto O R, Portillo A M, Aragón M A, Saboya M I, A de M P, Minero M A, et al. Prevalencia e intensidad de infección por geohelmintos y prevalencia de malaria en escolares de El Salvador. Biomédica. 2015 [Internet]. 2015 [consultado el 28 de diciembre de 2016]; 35 (3). Disponible en: https://www.revistabiomedica. org/index.php/biomedica/article/view/2408. 